\title{
Tuberculosis Pulmonar en Edad Pediátrica @(@)(
}

\author{
Pulmonary Tuberculosis in Pediatric Age
}

Teresa Anabel Lucín Yagual ${ }^{1}$, Yadira Elizabeth Yuz Humanante², Carlos David Villacreses Parrales ${ }^{3}$, \& Veronica Paulina Cáceres Manzano ${ }^{4}$

Recibido: 10-02-2019 / Revisado: 15-02-209 /Aceptado: 04-03-2019/ Publicado: 27-04-2019

\begin{abstract}
.
DOI: https://doi.org/10.33262/cienciadigital.v3i2.1.432

Mycobacterium tuberculosis infection in children under 15 years of age has an epidemiological and clinical behavior different from that of adults. We present the case of childhood tuberculosis, under 13 years of age that enters the unstable hospital unit with a reserved prognosis with respiratory insufficiency and caloric protein malnutrition, a negative smear microscopy is performed, a second GeneXpert test that is positive, he remains in an ICU isolated for 44 days, he received multiple antibiotic schemes including antifimic, evolves unfavorably to the applied therapies, dies of cardiorespiratory arrest. She had a family history, mother treated for 10 years of pulmonary tuberculosis, brother of the child presented pleural effusion 9 years ago, without diagnosis of TB, maternal grandmother with a history of TB. With what has been presented, it is evident the importance of establishing the necessary measures to optimize the early and adequate diagnosis of the different clinical forms of tuberculosis in children.
\end{abstract}

Keywords: Mycobacterium tuberculosis, smear microscopy, GeneXpert test, pulmonary tuberculosis, early diagnosis

\footnotetext{
1 Médico General; postgrado en Anestesiología, Hospital de Guasmo del MSP. techy2690@gmail.com ${ }^{2}$ Médico General; Maestría en Salud Ocupacional, Jefe Médico en Centro de Atención Ambulatoria Medicaldent. yadeliz_dasody@hotmail.com

${ }^{3}$ Médico General; Postgrado en Urología, Hospital de Ceibos del IESS; md.carlos.villacreses@gmail.com;

4 Abogada del tribunal de la República del Ecuador; Lcda. En Laboratorio Clínico Histopatológico; Especialista en Derecho, Mención Abogacía del Estado; verito17_caceres85@hotmail.com
} 


\section{Resumen.}

La infección por Mycobacterium tuberculosis en menores de 15 años tiene un comportamiento epidemiológico y clínico diferente al del adulto. Se presenta el caso de tuberculosis infantil, menor de 13 años de edad masculino que ingresa a la unidad hospitalaria inestable con pronóstico reservado con cuadro de insuficiencia respiratoria y desnutrición proteico calórica, se realiza baciloscopia que resulta negativa, una segunda prueba GeneXpert que resulta positiva; permanece en UCI aislado por 44 días, recibió múltiples esquemas de antibiótico incluido el antifímico, evoluciona desfavorablemente a las terapéuticas aplicadas, fallece de paro cardiorespiratorio. Tenía antecedentes familiares, madre tratada por 10 años de tuberculosis pulmonar, hermano del menor presento derrame pleural hace 9 años, sin diagnóstico de TB, la abuela materna con antecedentes de TB. Con lo presentado se evidencia la importancia de establecer las medidas necesarias para optimizar el diagnóstico precoz y adecuado de las distintas formas clínicas de tuberculosis en niños

Palabras claves: Mycobacterium tuberculosis, baciloscopia, prueba de GeneXpert, tuberculosis pulmonar, diagnostico precoz

\section{Introducción.}

La Tuberculosis (TB) continúa siendo un problema de salud pública, según datos de la Organización Mundial de la Salud, para el 2015 se estimó a nivel mundial 10.4 millones de casos nuevos en todas sus formas y se notificaron 6.1 millones. En Ecuador, la incidencia de tuberculosis (TBC) en la edad pediátrica es de 41.6/100 mil habitantes en menores de 15 años (2015). Con 8.400 casos nuevos. Este hecho puede explicar que a veces es difícil hacer el diagnóstico de TBC en pacientes pediátricos, sin embargo, fueron diagnosticados y notificados 5.215 casos, alcanzando una tasa de curación de $86 \%$. En 2016, zona 8 (Guayaquil, Durán y Samborondón) se diagnosticaron 2.386 casos de TB sensible, mientras que en la zona 5 (Guayas, Santa Elena, Los Ríos, Bolívar y Galápagos) hubo 919 casos reportados.

\section{Marco Teórico}

La tuberculosis es una enfermedad infecciosa causada por Mycobacterium tuberculosis complex (que engloba M. tuberculosis, M. bovis, M. africatum, M. canetti y M. microti), es un bacilo ácido-alcohol resistente (BAAR) y se considera el microorganismo que produce la gran mayoría de cuadros clínicos utiliza de reservorio el ser humano, tanto sano, como infectado y/o enfermo; aunque la infección se transmite, fundamentalmente, de pacientes enfermos bacilíferos (con baciloscopia positiva) por vía respiratoria al inhalar gotitas contaminadas. Otras vías de transmisión más infrecuentes son la cutáneo-mucosa, digestiva, uro-genital y transplacentaria. 
La tuberculosis es a menudo asintomática o poco expresiva, en ocasiones cursa con febrícula o fiebre prolongada, cansancio, anorexia y pérdida de peso, dependiendo de la forma de presentación podría aparecer también tos y dificultad respiratoria (neumonía, TB miliar) estridor o sibilancias (adenopatías mediastínicas, granuloma endobronquial), dolor en punta de costado con o sin fiebre (pleuresía), signos y síntomas de afectación del sistema nervioso central (meningitis, tuberculoma cerebral), adenopatía o adenopatías con mayor o menor expresión inflamatoria según la fase (adenitis); sinovitis de una articulación grande o más raramente pequeña (artritis), cifosis progresiva o dolor de espalda con o sin afectación de nervios periféricos (espondilitis); dolor abdominal o franca peritonitis (TB abdominal); hematuria o piuria estéril (TB renal y de vías urinarias); abscesos o úlceras cutáneas u otras manifestaciones de localizaciones infrecuentes; signos de hipersensibilidad tuberculínica, como eritema nodoso o conjuntivitis flictenular.

La presentación de la tuberculosis en la mayoría de niños infectados están asintomáticos o presentan clínica inespecífica como fiebre, astenia, artralgias, etc; aunque otras veces puede existir clínica pulmonar como tos, dificultad respiratoria, expectoración y/o dolor torácico; siendo la localización pulmonar la forma más frecuente de la enfermedad en niños y, dentro de ella, la ganglionar; aunque, a menor edad del niño, existe mayor riesgo de presentar formas extrapulmonares como la meningoencefalitis y diseminadas o linfohematógenas con elevada morbimortalidad.

El riesgo de desarrollar tuberculosis es mucho mayor en adictos o usuarios de drogas intravenosas, personas quienes trabajen o vivan en hacinamiento (cárceles, hogares de cuidado crónico), los provenientes de zonas de alta incidencia, la co-infección con VIH y otros estados de inmunosupresión tales como trasplante de órganos, diabéticos y enfermedad renal crónica. Se ha considerado también por algunos autores el tabaquismo como factor de riesgo. Actualmente, el incremento en el uso de medicamentos como los antagonistas del factor de necrosis tumoral (FNT) en enfermedades como la artritis reumatoide (AR), psoriasis y enfermedad de Crohn, han aumentado la incidencia de infección por M. tuberculosis, especialmente las formas extra pulmonares y diseminada . En el grupo de pacientes con inmunosupresión de base y especialmente en pacientes con infección por VIH que tengan infección latente, el riesgo de desarrollar enfermedad, y en especial formas diseminadas o extra pulmonares, se incrementa varias veces, con un riesgo anual mayor que en el inmuno competente.

\section{Metodologia.}

El presente estudio es de tipo descriptivo que involucra paciente hospitalizado de sexo masculino 13 años de edad con antecedentes de madre tuberculosis pulmonar curada hace 9 meses, hermano del menor presento derrame pleural hace 9 años, sin diagnóstico de TB, la abuela materna con antecedentes de $\mathrm{TB}$, sub diagnosticado en 2 unidades operativas. Ingresa a unidad hospitalaria de segundo nivel con insuficiencia respiratoria, requerimiento 
de oxígeno, con talla y peso bajos, perdida de panículo adiposo, llenado capilar lento, ganglios cervicales en región cervical, axilar izquierda, pérdida de fuerza muscular, a la auscultación crepitantes bilaterales, disminución del Murmullo Vesicular, basal derecha. Que inicio tratamiento antifimico 4 días previos al ingreso y baciloscopia negativa de inicio. Se realiza segunda baciloscopia y la prueba de GeneXpert con resultado positivo, en donde se solicita el traslado a una unidad de especialidad de tercer nivel, pero no se encontró disponibilidad.

EVOLUCIÓN: paciente ingresa en condiciones clínicas inestables, pronóstico reservado, con un déficit nutricional del $45 \%$, gran afectación pulmonar, abundantes infiltrados bilaterales (fig. 1), bulla enfisematosa, cuadro de insuficiencia respiratorio aguda, Que debuta posteriormente con neumotórax a repetición (durante su estancia hospitalaria). Tratado con amplios esquemas antibióticos, antifimico y antifúngicos, pero que, debido a sus múltiples comorbilidades, inmunodepresión, larga estancia hospitalaria, contaminación intrahospitalaria, no respondió favorablemente a las terapias indicadas, progresa a entubación endotraqueal, y paro cardiorespiratorio.

Figura.1: Rx. de torax infiltrado algodonoso en panal de abeja (patrón miliar)

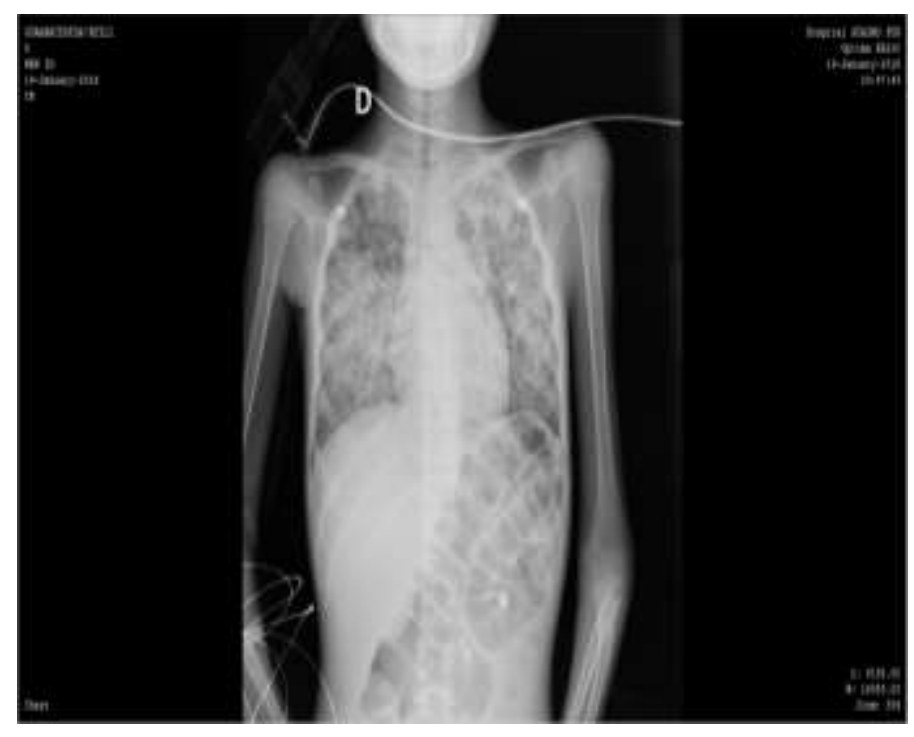

\section{Resultados.}

Estuvo hospitalizado 44 dias en el área de UCI adulto aislado de los demás pacientes, durante su estancia hospitalaria, se realiza segunda baciloscopia y la prueba de GeneXpert cuyos resultados fueron negativo y de baja sensibilidad, el examen de biometría hemática evidencia Anemia. Se realizaban controles de laboratorio incluida gasometría y electrolitos que revelan hiponatremia e hipocalcemia, no se indica los motivos por los cuales dias 
posteriores se suspenden los controles de biometría gasometría y electrolitos, se le realiza tomografía de tórax en donde se observa cavernas apicales y patrón miliar bilateral (fig. 2) y se realizaron cambios de esquemas de antibiótico, antifimicos sin evidencia de mejoría clínica, se instaura tratamiento antimicótico de acuerdo al esquema y se insiste en la necesidad del traslado del paciente a una entidad de tercer nivel para continuar tratamiento multidisciplinario pero no se da por falta de espacio físico, por lo que se complica con neumonía nosocomial y neumotórax por lo que fallece.

El menor convivía con familiares recientemente tratados de tuberculosis siendo de bajos recursos económicos y de zonas marginales ya reflejaban los factores que determinan alto riesgo de infección, por este motivo se debería de empezar a realizar una prueba de tuberculina a todo niño con estos precedentes. Tras su realización se debe actuar en consecuencia del resultado obtenido pautando profilaxis tras descartar enfermedad o tratando el caso.

Figura 2: tac: imagenes reticulonodulares bilaterales tipo cavernas

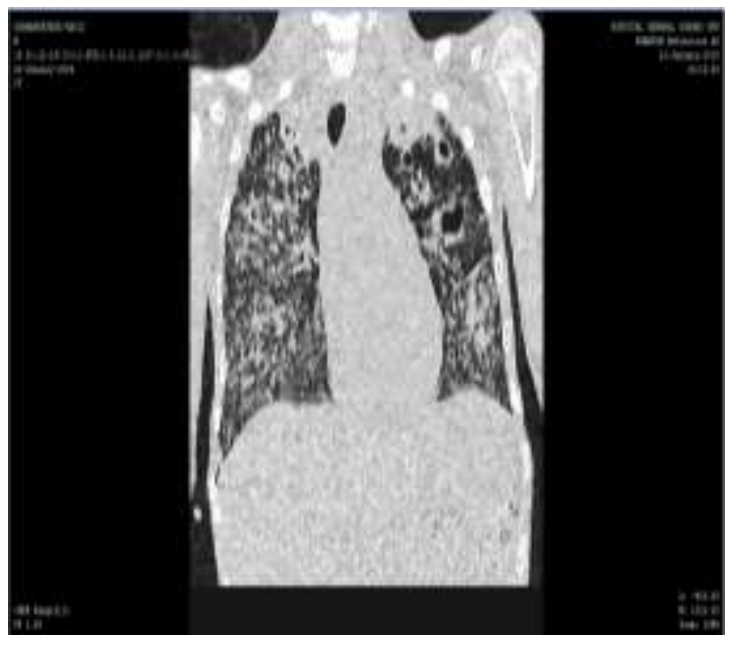

\section{Conclusiones.}

- La OMS (Organización Mundial de la Salud) estima que 4.000 personas fallecen al día por la tuberculosis; más del $95 \%$ de las víctimas están en países con ingresos bajos donde hay poco o nulo acceso al tratamiento con antibióticos, se estima que, en el 2016, se enfermaron de tuberculosis, un millón de niños (de 0 a 14 años) de 250.000 (incluyendo con VIH), murieron debido a estas causas.

- En la actualidad estudios realizados revelan que la tuberculosis infantil está íntimamente ligada a la del adulto y se puede considerar que los casos pediátricos son los centinelas de la comunidad; de ahí la importancia del diagnóstico, clasificación, aislamiento y terapia adecuada de los enfermos, así como el correcto tratamiento de la exposición y la infección tuberculosa para modificar la situación 
epidemiológica de la tuberculosis en cada país, siendo fundamental la unificación de los criterios de las técnicas de los medios diagnostico además de actualizar los protocolos de actuación en todas las entidades hospitalarias.

\section{Referencias bibliográficas.}

D. Moreno-Pérez ${ }^{\mathrm{a}}$, A. Andrés Martín ${ }^{\mathrm{b}, \mathrm{c}, \mathrm{d}, \mathrm{n}}$, N. Altet Gómez ${ }^{\mathrm{e}, \mathrm{f}, \mathrm{n}}, \mathrm{F}$. Baquero-Artigao ${ }^{\mathrm{g}, \mathrm{n}}, \mathrm{A}$. Escribano Montaner ${ }^{\mathrm{h}, \tilde{n}}$, D. Gómez-Pastrana Durán ${ }^{\mathrm{i}, \tilde{n}}$, R. González Montero ${ }^{\mathrm{j}, \mathrm{n}}$, M.J. Mellado Peña $^{\mathrm{k}, \mathrm{n}}$, C. Rodrigo-Gonzalo-de-Liria ${ }^{\text {l,n }}$, M.J. Ruiz Serrano ${ }^{\mathrm{m}}$

Unidad de Infectología e Inmunodeficiencias, Servicio de Pediatría, Hospital MaternoInfantil Carlos Haya, Universidad de Málaga, Málaga, España

Unidad de Neumología y Alergia Pediátrica, Servicio de Pediatría, Hospital Universitario Virgen de Valme, Sevilla, España

Organización Mundial de la Salud (OMS). Global tuberculosis control: wHO report 2010. Disponible en: http://www.who. int/tb/publications/global_report/2010/gtbr10_main.pdf 2.

Rodríguez Valín E, Garrido Estepa M, Villarrubia Enseñat S, et al. Epidemiología de la tuberculosis pediátrica en España, 2005-2009. Rev Esp Salud Pública. 2012; 85: 49-59.

Revista Española de Clínica e Investigación. Enero - Febrero 2016, Volumen 72 - Número 1, Director Manuel Hernández Rodríguez, Secretario de redaccion Arturo Muñoz Villa

Prevención, diagnóstico, tratamiento y control de la tuberculosis Guía de Práctica Clínica, Segunda edición 2018, Ministerio de Salud Pública.

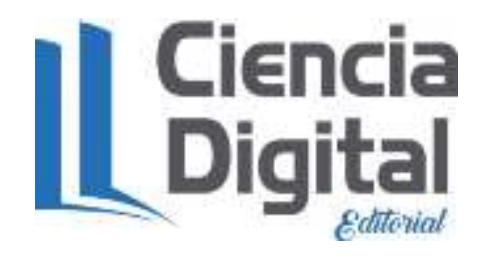




\section{PARA CITAR EL ARTÍCULO INDEXADO.}

Lucín Yagual, T., Yuz Humanante, Y., Villacreses Parrales, C., \& Cáceres Manzano, V. (2019). Tuberculosis pulmonar en edad pediátrica. Ciencia Digital, 3(2.1), 78-84. https://doi.org/10.33262/cienciadigital.v3i2.1.432

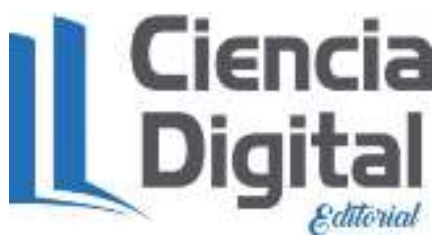

El artículo que se publica es de exclusiva responsabilidad de los autores y no necesariamente reflejan el pensamiento de la Revista Ciencia Digital.

El artículo queda en propiedad de la revista y, por tanto, su publicación parcial y/o total en otro medio tiene que ser autorizado por el director de la Revista Ciencia Digital.
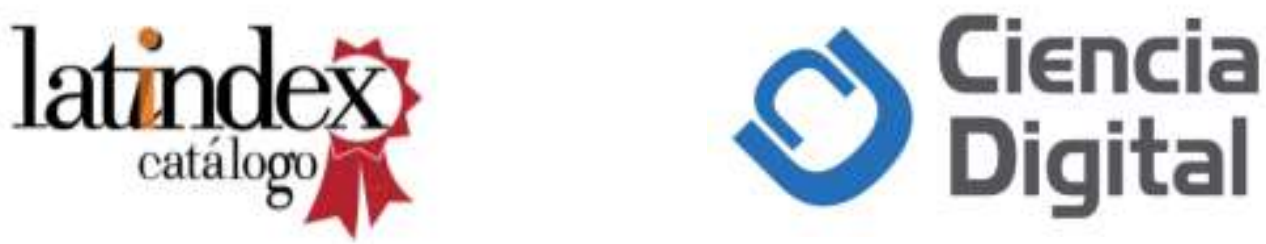